First published in:

JOURNAL DE PHYSIQUE IV, 105, 167-174, 2003

\title{
Crystallographic Texture Induced Anisotropy in Copper: An Approach Based on a Tensorial Fourier Expansion of the CODF
}

\author{
Thomas Böhlke and Albrecht Bertram \\ Otto-von-Guericke-University of Magdeburg \\ Institute of Mechanics, Postfach 4120 \\ 39016 Magdeburg, Germany
}

\begin{abstract}
We consider a tensorial Fourier expansion of the crystallite orientation distribution function for aggregates of cubic crystals. The coefficient of rank four is determined explicitly. It is shown that this coefficient governs the anisotropic bounds of the linear elastic behavior of polycrystals. Recently, a phenomenological model has been proposed [4, 6], that describes the evolving elastic anisotropy in copper. The model also reproduces the cyclic Swift effect, which is due to a texture induced plastic anisotropy [7]. In the model the anisotropic part of the effective elastic stiffness tensor is used to define a texture dependent quadratic yield function. The present paper gives a new interpretation of the aforementioned model in terms of an approximation of the crystallographic texture by means of a 4th-order coefficient of the tensorial Fourier expansion of the crystallite orientation distribution function.
\end{abstract}

\section{Introduction}

On the macroscale the mechanical behavior of polycrystalline metals is generally governed by the crystallographic texture, the morphology of the grains, and the hardening state in the aggregate. In the present work we restrict the investigation to the macroscopic anisotropy due to an inhomogeneous crystallite orientation distribution function (CODF), i.e., the crystallographic texture.

First, a tensorial Fourier expansion of the CODF is discussed in detail for aggregates of cubic crystals. The coefficients of this expansion can be considered as internal variables for models, which take into account the crystallographic texture. The most simple internal variable in the aforementioned sense is the first coefficient of the expansion. Such an approach approximates the CODF by a 4th-order moment tensor. This moment tensor is derived in detail in the sequel and its properties are discussed. Then the macroscopic elastic anisotropy is considered. It is shown that the 4th-order moment tensor governs the anisotropic part of all common types of orientation averages like, e.g., the Voigt or Reuss estimates. Furthermore, experiments and theoretical considerations show that the anisotropy of the plastic behavior of metals is correlated with the elastic anisotropy [15, 16]. Therefore, the anisotropy of the plastic behavior can be estimated by means of the elastic anisotropy for moderately textured 
polycrystals. A simple form of a yield condition, although of limited applicability, is given by a quadratic form of the stresses $[22,13,21]$. We show, how the texture information can be incorporated into a quadratic yield condition. It can be shown that such a model allows for predicting the plastic anisotropy of rolled sheets and the cyclic Swift effect, which can be observed in torsion experiments $[4,6,7]$.

\section{Tensorial Representation of the CODF}

Homogenization Based on Orientation Averages. It is assumed that the distribution of crystal orientations represents the dominant aspect of microstructure. This assumption is reasonable if the two-point statistics of crystal orientations are isotropic [2]. The orientation of a crystallite can be described by a proper orthogonal tensor $\boldsymbol{Q} \in$ Orth $^{+}$

$$
\boldsymbol{Q}=\boldsymbol{g}_{i} \otimes \boldsymbol{e}_{i}
$$

where $\left\{\boldsymbol{e}_{i}\right\}$ and $\left\{\boldsymbol{g}_{i}\right\}$ represent a orthonormal basis fixed to the sample and to the (orthonormal) lattice vectors of the crystal, respectively. The components of $\boldsymbol{Q}$ with respect to $\left\{\boldsymbol{e}_{i}\right\}$ are given by the directional cosines

$$
Q_{i j}=\boldsymbol{e}_{i} \cdot \boldsymbol{Q} \boldsymbol{e}_{j}=\boldsymbol{e}_{i} \cdot \boldsymbol{g}_{j}
$$

The $k$-th row of the matrix $Q_{i j}$ contains the components of $\boldsymbol{g}_{k}$ with respect to $\left\{\boldsymbol{e}_{i}\right\}$. The $k$-th column of the matrix $Q_{i j}$ contains the components of $\boldsymbol{e}_{k}$ with respect to $\left\{\boldsymbol{g}_{i}\right\}$.

The distribution of crystal orientations $\boldsymbol{Q}$ can be quantitatively described by the orientation distribution function $f(\boldsymbol{Q})[8,19]$. It represents the one-point statistics of crystal orientations. The function $f(\boldsymbol{Q})$ specifies the volume fraction $\mathrm{d} v / v$ of crystals having the orientation $\boldsymbol{Q}$, i.e.

$$
\frac{\mathrm{d} v(\boldsymbol{Q})}{v}=f(\boldsymbol{Q}) \mathrm{d} Q
$$

$\mathrm{d} Q$ is the volume element in $\operatorname{Orth}^{+}$which ensures an invariant integration over Orth $^{+}$[11], i.e.

$$
\int_{S O(3)} f(\boldsymbol{Q}) \mathrm{d} Q=\int_{S O(3)} f\left(\boldsymbol{Q} \boldsymbol{Q}_{0}\right) \mathrm{d} Q \quad \forall \boldsymbol{Q}_{0} \in \operatorname{Orth}^{+}
$$

If $\mathrm{Orth}^{+}$is parameterized by Euler angles, the volume element $\mathrm{d} Q$ is given by

$$
\mathrm{d} Q=\frac{\sin (\Phi)}{8 \pi^{2}} \mathrm{~d} \varphi_{1} \mathrm{~d} \Phi \mathrm{d} \varphi_{2}
$$

The function $f(\boldsymbol{Q})$ is nonnegative and normalized such that

$$
f(\boldsymbol{Q}) \geq 0 \quad \forall \boldsymbol{Q} \in \text { Orth }^{+}, \quad \int_{S O(3)} f(\boldsymbol{Q}) \mathrm{d} Q=1
$$

Let be $\psi(\boldsymbol{Q}(\boldsymbol{x}))$ a mechanical quantity of a crystallite which depends on the position vector only by its dependence on the orientation $\boldsymbol{Q}$. Volume averages of such a quantity can be transformed by 
equation (3) into averages over $\operatorname{Orth}^{+}$

$$
\bar{\psi}=\frac{1}{v} \int_{v} \psi(\boldsymbol{Q}(\boldsymbol{x})) \mathrm{d} v=\int_{S O(3)} f(\boldsymbol{Q}) \psi(\boldsymbol{Q}) \mathrm{d} Q .
$$

The orientation distribution function $f(\boldsymbol{Q})$ reflects both the symmetry of the crystallites forming the aggregate and the sample symmetry, which results from processing. The crystal symmetry implies the following symmetry relation for $f(\boldsymbol{Q})$

$$
f(\boldsymbol{Q})=f\left(\boldsymbol{Q} \boldsymbol{H}^{C}\right) \quad \forall \boldsymbol{H}^{C} \in S^{C} \subseteq O r t h^{+} .
$$

$S^{C}$ denotes the symmetry group of the crystallite. The sample symmetry implies the following symmetry relation for $f(\boldsymbol{Q})$

$$
f(\boldsymbol{Q})=f\left(\boldsymbol{H}^{S} \boldsymbol{Q}\right) \quad \forall \boldsymbol{H}^{S} \in S^{S} \subseteq O r t h^{+}
$$

[25]. $S^{S}$ denotes the symmetry group of the sample.

Tensorial Fourier Expansion of the CODF. For the subsequent considerations it is assumed that the CODF is square integrable. This property implies the existence of a tensorial Fourier expansion. Adams et al. [1] and Guidi et al. [12] considered this expansion for the special case of a cubic crystal symmetry. Zheng and $\mathrm{Fu}[24,25]$ analyzed the expansion for arbitrary crystal and sample symmetries. For aggregates of cubic crystals the Fourier expansion has the form

$$
f(\boldsymbol{Q})=1+f_{4}(\boldsymbol{Q})+f_{6}(\boldsymbol{Q})+f_{8}(\boldsymbol{Q})+\ldots,
$$

where

$$
f_{i}=\mathbb{V}_{\langle i\rangle}^{\prime} \cdot \mathbb{F}_{\langle i\rangle}^{\prime}(\boldsymbol{Q}), \quad \quad \mathbb{F}_{\langle i\rangle}^{\prime}(\boldsymbol{Q})=\boldsymbol{Q} \star \mathbb{T}_{\langle i\rangle}^{\prime} .
$$

The coefficients of this expansion $\mathbb{V}_{\langle i\rangle}^{\prime}$ and $\mathbb{T}_{\langle i\rangle}^{\prime}$ are completely symmetric and traceless tensors of even rank $i$. E.g., in the case of $\mathbb{V}^{\prime}=\mathbb{V}_{\langle 4\rangle}^{\prime}$ the following relations hold

$$
V_{i j k l}^{\prime}=V_{j i k l}^{\prime}=V_{k l i j}^{\prime}=V_{k j i l}^{\prime}=\ldots, \quad V_{i i k l}^{\prime}=0 .
$$

Completely symmetric and traceless tensors are called irreducible (indicated by a prime). The star in equation $(11)_{2}$ denotes the Rayleigh product, which for 4 th-order tensors $\mathbb{V}=V_{i j k l} \boldsymbol{e}_{i} \otimes \boldsymbol{e}_{j} \otimes \boldsymbol{e}_{k} \otimes \boldsymbol{e}_{l}$ is defined by

$$
\boldsymbol{Q} \star \mathbb{V}=V_{i j k l}\left(\boldsymbol{Q} \boldsymbol{e}_{i}\right) \otimes\left(\boldsymbol{Q} \boldsymbol{e}_{j}\right) \otimes\left(\boldsymbol{Q} \boldsymbol{e}_{k}\right) \otimes\left(\boldsymbol{Q} \boldsymbol{e}_{l}\right) .
$$

Higher rank Rayleigh products are defined analogously. From equations (8), (9), (10), and (11) it can be concluded that the $\mathbb{T}_{\langle i\rangle}^{\prime}$ reflect the crystal symmetry as

$$
f(\boldsymbol{Q})=f\left(\boldsymbol{Q} \boldsymbol{H}^{C}\right) \quad \forall \boldsymbol{H}^{C} \in S^{C} \Rightarrow \mathbb{T}_{\langle i\rangle}^{\prime}=\boldsymbol{H}^{C} \star \mathbb{T}_{\langle i\rangle}^{\prime} \quad \forall \boldsymbol{H}^{C} \in S^{C},
$$

whereas the $\mathbb{V}_{\langle i\rangle}^{\prime}$ have the sample symmetry

$$
f(\boldsymbol{Q})=f\left(\boldsymbol{H}^{S} \boldsymbol{Q}\right) \quad \forall \boldsymbol{H}^{S} \in S^{S} \quad \Rightarrow \quad \mathbb{V}_{\langle i\rangle}^{\prime}=\boldsymbol{H}^{S} \star \mathbb{V}_{\langle i\rangle}^{\prime} \quad \forall \boldsymbol{H}^{S} \in S^{S} .
$$


The orthogonality relation

$$
\int_{S O(3)} \mathbb{F}_{\langle i\rangle}^{\prime}(\boldsymbol{Q}) \otimes \mathbb{F}_{\langle j\rangle}^{\prime}(\boldsymbol{Q}) \mathrm{d} Q=0 \quad \forall i \neq j
$$

allows for computing the coefficients $\mathbb{V}_{\langle i\rangle}^{\prime}$ of the Fourier expansion (10) for a given function $f(\boldsymbol{Q})$. A combination of equations (10) and (16) gives a linear system of equations for the determination of each $\mathbb{V}_{\langle i\rangle}^{\prime}$

$$
\mathbb{J}_{\langle i\rangle}^{\prime}=\mathbb{E}_{\langle 2 i\rangle}\left[\mathbb{V}_{\langle i\rangle}^{\prime}\right]
$$

where

$$
\mathbb{J}_{\langle i\rangle}^{\prime}=\int_{S O(3)} f(\boldsymbol{Q}) \mathbb{F}_{\langle i\rangle}^{\prime}(\boldsymbol{Q}) \mathrm{d} Q
$$

and

$$
\mathbb{E}_{\langle 2 i\rangle}=\int_{S O(3)} \mathbb{F}_{\langle i\rangle}^{\prime}(\boldsymbol{Q}) \otimes \mathbb{F}_{\langle i\rangle}^{\prime}(\boldsymbol{Q}) \mathrm{d} Q
$$

The Coefficient $\mathbb{V}_{\langle 4\rangle}^{\prime}$. For the formulation of macroscopic material models, which describe the generally anisotropic viscoplastic behavior of metals, internal variables are commonly used. In order to take into account the crystallographic texture, it is reasonable to consider the coefficients $\mathbb{V}_{\langle i\rangle}^{\prime}$ as internal variables, which can be determined by texture measurements. The linear space $S_{y m^{\prime}}{ }_{k}$ of irreducible tensors of rank $k$ has the dimension

$$
\operatorname{dim}\left(\operatorname{Sym}_{k}^{\prime}\right)=2 k+1
$$

[11]. Hence, the number of independent components of the $\mathbb{V}_{\langle i\rangle}^{\prime}$ increases only linearly with the tensor rank. The most simple phenomenological model, that takes into account the crystallographic texture, would be based on $\mathbb{V}_{\langle 4\rangle}^{\prime}$.

As an example, the determination of $\mathbb{V}^{\prime}=\mathbb{V}_{\langle 4\rangle}^{\prime}$ is discussed in detail for the case of a cubic crystal symmetry. In the following, we drop the index denoting the tensor rank if forth-order tensors are concerned. For the computation of $\mathbb{V}^{\prime}$ it is helpful to consider first the CODF of a single cubic crystal having the orientation $\boldsymbol{Q}_{0}$. The CODF is given by the Dirac distribution, i.e. $f(\boldsymbol{Q})=\delta\left(\boldsymbol{Q}, \boldsymbol{Q}_{0}\right)$. Orientation averages are trivial in this case and yield the single crystal property

$$
\bar{\psi}=\int_{S O(3)} f(\boldsymbol{Q}) \psi(\boldsymbol{Q}) \mathrm{d} Q=\int_{S O(3)} \delta\left(\boldsymbol{Q}, \boldsymbol{Q}_{0}\right) \psi(\boldsymbol{Q}) \mathrm{d} Q=\psi\left(\boldsymbol{Q}_{0}\right) .
$$

In a similar way one obtains from equation (18) with $\mathbb{J}^{\prime}=\mathbb{J}_{\langle 4\rangle}^{\prime}$ and $\mathbb{F}^{\prime}(\boldsymbol{Q})=\mathbb{F}_{\langle 4\rangle}^{\prime}(\boldsymbol{Q})$

$$
\mathbb{J}_{C}^{\prime}=\mathbb{J}^{\prime}=\int_{S O(3)} f(\boldsymbol{Q}) \mathbb{F}^{\prime}(\boldsymbol{Q}) \mathrm{d} Q=\mathbb{F}^{\prime}\left(\boldsymbol{Q}_{0}\right)=\boldsymbol{Q}_{0} \star \mathbb{T}^{\prime} .
$$

The crucial point for the determination of $\mathbb{V}_{C}^{\prime}$ is the fact that the linear system (17) can be reduced to a scalar equation. Since a single crystal is considered, the crystal and sample symmetry coincide. As a result, $\mathbb{V}^{\prime}$ has the same symmetry as $\mathbb{T}^{\prime}$ and $\mathbb{J}^{\prime}$. Furthermore, definition (19) implies that $\mathbb{E}_{\langle 2 i\rangle}$ is an isotropic tensor, which acts on the $i$-th order irreducible tensors. Hence, the following relation holds

$$
\mathbb{V}_{C}^{\prime}=\beta \mathbb{J}_{C}^{\prime}=\beta \boldsymbol{Q}_{0} \star \mathbb{T}^{\prime}
$$


From equation (17) one deduces

$$
\mathbb{J}_{C}^{\prime}=\mathbb{E}_{\langle 8\rangle}\left[\mathbb{V}_{C}^{\prime}\right]=\beta \mathbb{E}_{\langle 8\rangle}\left[\mathbb{J}_{C}^{\prime}\right]
$$

and

$$
\mathbb{J}_{C}^{\prime} \cdot \mathbb{J}_{C}^{\prime}=\beta \mathbb{J}_{C}^{\prime} \cdot \mathbb{E}_{\langle 8\rangle}\left[\mathbb{J}_{C}^{\prime}\right] .
$$

The last equation is not changed if we set $\boldsymbol{Q}_{0}=\boldsymbol{I}$, i.e. $\mathbb{J}_{C}^{\prime}=\mathbb{T}^{\prime}$. Hence $\beta$ is given by

$$
\beta=\frac{\mathbb{T}^{\prime} \cdot \mathbb{T}^{\prime}}{\mathbb{T}^{\prime} \cdot \mathbb{E}_{\langle 8\rangle}\left[\mathbb{T}^{\prime}\right]}=\frac{\mathbb{T}^{\prime} \cdot \mathbb{T}^{\prime}}{\int_{S O(3)}\left(\mathbb{T}^{\prime} \cdot\left(\boldsymbol{Q} \star \mathbb{T}^{\prime}\right)\right)^{2} \mathrm{~d} Q}
$$

The tensor $\mathbb{T}^{\prime}$, which is irreducible and of cubic symmetry, is given by

$$
\mathbb{T}^{\prime}=\frac{\sqrt{30}}{30} \alpha(5 \mathbb{D}-\boldsymbol{I} \otimes \boldsymbol{I}-2 \mathbb{I}), \quad \mathbb{D}=\sum_{i=1}^{3} \boldsymbol{e}_{i} \otimes \boldsymbol{e}_{i} \otimes \boldsymbol{e}_{i} \otimes \boldsymbol{e}_{i}, \quad\left\|\mathbb{T}^{\prime}\right\|=|\alpha| .
$$

[6]. $\boldsymbol{I}$ and $\mathbb{I}$ denote the 2nd-order identity tensor and the 4th-order identity tensor on symmetric 2nd-order tensors, respectively. $\left\|\mathbb{T}^{\prime}\right\|$ is the Frobenius norm defined by $\left\|\mathbb{T}^{\prime}\right\|^{2}=\mathbb{T}^{\prime} \cdot \mathbb{T}^{\prime}=T_{i j k l}^{\prime} T_{i j k l}^{\prime}$. A straight forward calculation yields

$$
\beta=\frac{9}{|\alpha|^{2}} .
$$

The CODF of a number of $\kappa$ discrete single crystals with orientations $\boldsymbol{Q}_{\gamma}$ and volume fractions $\nu_{\gamma}$ is

$$
f(\boldsymbol{Q})=\sum_{\gamma=1}^{\kappa} \nu_{\gamma} f_{\gamma}(\boldsymbol{Q})
$$

with $f_{\gamma}(\boldsymbol{Q})=\delta\left(\boldsymbol{Q}, \boldsymbol{Q}_{\gamma}\right)$. Applying the Fourier expansion to each of the $f_{\gamma}$ gives the coefficients $\mathbb{V}_{\gamma}^{\prime}$. The coefficient $\mathbb{V}^{\prime}$ with respect to $f(\boldsymbol{Q})$ is then obtained by

$$
\mathbb{V}^{\prime}=\sum_{\gamma=1}^{\kappa} \nu_{\gamma} \mathbb{V}_{\gamma}^{\prime}
$$

or, due to the assumed equivalence of volume and orientational averages, by

$$
\mathbb{V}^{\prime}=\int_{S O(3)} f(\boldsymbol{Q}) \mathbb{V}_{C}^{\prime}(\boldsymbol{Q}) \mathrm{d} Q
$$

Equations (23), (28), and (31) allows for calculating the 4th-order coefficient of the tensorial Fourier expansion of the CODF of an aggregate of cubic crystals. It is reasonable to choose the scalar $\alpha$ in such a way that the Frobenius norm of the coefficient $\mathbb{V}^{\prime}$ is equal to one for a single crystal and in $[0,1)$ for a polycrystal. Then the amount of anisotropy can be estimated easily by means of $\left\|\mathbb{V}^{\prime}\right\|$. A simple calculation yields $\left\|\mathbb{V}_{C}^{\prime}\right\|=9 / \alpha$. Hence, the aforementioned normalization is obtained by setting $\alpha=9$. Finally, the 4 th-order moment tensor is given by

$$
\mathbb{V}^{\prime}(\alpha=9)=\frac{\sqrt{30}}{30}\left(5 \int_{S O(3)} \sum_{i=1}^{3} f(\boldsymbol{Q}) \boldsymbol{g}_{i} \otimes \boldsymbol{g}_{i} \otimes \boldsymbol{g}_{i} \otimes \boldsymbol{g}_{i} \mathrm{~d} Q-\boldsymbol{I} \otimes \boldsymbol{I}-2 \mathbb{I}\right) .
$$


The tensor $\mathbb{V}^{\prime}$ has the following properties

- $\mathbb{V}^{\prime}$ is irreducible, see equation (12), and has nine independent components,

- $\mathbb{V}^{\prime}$ has the sample symmetry and covers all possible symmetries (e.g., a triclinic, orthotropic, transverse isotropic, or isotropic symmetry)

- $\left\|\mathbb{V}^{\prime}\right\|=0$ for an isotropic polycrystal,

- $\left\|\mathbb{V}^{\prime}\right\| \in(0,1)$ for a textured polycrystal,

- $\left\|\mathbb{V}^{\prime}\right\|=1$ for a single crystal.

\section{Macroscopic Elastic Properties}

The most simple orientation averages are the arithmetic and the harmonic mean of the local stiffness tensors $\mathbb{C}$ with $\mathbb{C}^{-1}=\mathbb{S}$, which where first suggested by Voigt and Reuss

$$
\mathbb{C}^{V}=\int_{S O(3)} f(\boldsymbol{Q}) \mathbb{C}(\boldsymbol{Q}) \mathrm{d} Q, \quad \mathbb{S}^{R}=\int_{S O(3)} f(\boldsymbol{Q}) \mathbb{S}(\boldsymbol{Q}) \mathrm{d} Q
$$

$[23,18]$. The arithmetic mean and the harmonic mean correspond to the assumption of homogeneous strain and stress fields, respectively. These approaches give upper and lower bounds for the strain energy density. The lower the degree of the elastic anisotropy on the microscale, the more accurate the averages are. An approach that focuses on a homogenization resulting in unique effective properties, such that the inverse of the mean compliance is equal to the mean stiffness, was given by Aleksandrov and Aisenberg [3] and further developed by Matthies and Humbert [17]. Here, the geometric mean of the local elastic moduli is used

$$
\mathbb{C}^{A}=\exp \left(\int_{S O(3)} f(\boldsymbol{Q}) \ln (\mathbb{C}(\boldsymbol{Q})) \mathrm{d} Q\right), \quad \mathbb{S}^{A}=\exp \left(\int_{S O(3)} f(\boldsymbol{Q}) \ln (\mathbb{S}(\boldsymbol{Q})) \mathrm{d} Q\right) \equiv \mathbb{C}^{A^{-1}} .
$$

Predictions based on this approach are similar to self-consistent estimates and close to experimental data [17]. Orientation averages of stiffnesses or compliances with a cubic symmetry generally allow for the following unique decomposition of the effective properties

$$
\begin{aligned}
& \mathbb{C}^{V}=\mathbb{C}^{V I}+\xi^{V} \mathbb{V}^{\prime}, \\
& \ln \left(\mathbb{C}^{A}\right)=\ln \left(\mathbb{C}^{A I}\right)+\xi^{A} \mathbb{V}^{\prime} \\
& \mathbb{S}^{R}=\mathbb{S}^{R I}+\xi^{R} \mathbb{V}^{\prime}
\end{aligned}
$$

$[5,6]$. The first terms on the right-hand sides represent the isotropic parts of the average and the second terms the purely anisotropic parts. The isotropic parts are independent of the texture in the aggregate. $\mathbb{C}^{V I}$ and $\mathbb{S}^{R I}$ have been determined by Voigt and Reuss. The anisotropic part vanishes for an uniform orientation distribution. On the one hand, the anisotropic parts are influenced by the degree of anisotropy of the single crystals through the scalars $\xi^{V}, \xi^{A}, \xi^{R}$, which depend only on the eigenvalues of the single crystal stiffness, on the other hand, by the orientation distribution in terms 
of the 4 th-order moment tensor $\mathbb{V}^{\prime}$. It is remarkable that the direction of the purely anisotropic part is independent of the type of averaging.

\section{Macroscopic Plastic Properties}

There is experimental evidence that the effective elastic and (visco)plastic properties are correlated for textured polycrystals $[9,20,14,10]$. Therefore, the anisotropy of the (visco)plastic behavior (e.g., the average plastic strain ratio, the in-plane anisotropy) can be inferred not only from destructive tests, but also from non-destructive measurements of the elastic anisotropy parameters. Based on these findings, Man [15, 16] formulated a quadratic yield function, which assumes that the elastic and plastic properties are correlated linearly. As a result, the anisotropic part of the effective elasticity tensors specifies the anisotropic part of the quadratic yield condition. The introduction of a quadratic yield function goes back to v. Mises [22], who introduced a general 4th-order tensor of plastic moduli that establishes a quadratic yield condition in terms of stresses. Man assumed his correlation for the special case of an orthotropic sample symmetry. This approach is generalized by assuming that the anisotropic part of the quadratic yield condition is governed by the 4th-order tensor $\tilde{\mathbb{A}}^{\prime}$ with respect to the intermediate configuration. Then the yield condition is given by

$$
\Delta\left(\tilde{\boldsymbol{S}}^{\prime}, \tilde{\mathbb{V}}^{\prime}\right)=\tilde{\boldsymbol{S}}^{\prime} \cdot \tilde{\mathbb{N}}\left(\tilde{\mathbb{V}}^{\prime}\right)\left[\tilde{\boldsymbol{S}}^{\prime}\right]-\frac{2}{3} \sigma_{F}^{2}=0
$$

where $\tilde{\mathbb{N}}\left(\tilde{\mathbb{V}}^{\prime}\right)=\mathbb{P}_{2}^{I}+\eta \tilde{\mathbb{V}}^{\prime} . \tilde{\boldsymbol{S}}^{\prime}$ is the 2.Piola-Kirchhoff stress tensor with respect to the intermediate configuration. For $\tilde{\mathbb{V}}^{\prime}=\mathbb{O}$, i.e. an nontextured aggregate, the classical isotropic v.Mises yield condition is obtained. The tensor $\tilde{\mathbb{N}}$ has to be positive definite. This requirement constraints the scalar $\eta$. The influence of the crystallographic texture on the macroscopic yield loci has been taken into account by a constant 4th-order tensor by v. Mises [22], Hill [13], Teodosiu [21]. Such a tensor is specified by the suggested approach up to one scalar $\eta$.

Single crystalline copper exhibits a strong anisotropy of both the elastic and the viscoplastic behavior. This fact causes both the effective elastic and the viscoplastic properties of the polycrystal to become anisotropic if a crystallographic texture evolves. It can be shown that a model, which takes into account the crystallographic texture by means of the moment tensor $\mathbb{V}^{\prime}$, allows for predicting the plastic anisotropy of rolled copper sheets and the cyclic Swift effect, which can be observed in torsion experiments $[4,6,7]$.

\section{References}

[1] B.L. Adams, J.P. Boehler, M. Guidi, and E.T. Onat. Group theory and representation of microstructure and mechanical behavior of polycrystals. J. Mech. Phys. Solids, 40(4):723-737, 1992.

[2] B.L. Adams, T.A. Mason, T. Olson, and D.D. Sam. Theory of grain boundary structure effects on mechanical behavior. Materials Science Forum, 157-162:1731-1738, 1994.

[3] K.S. Aleksandrov and L.A. Aisenberg. A method of calculating the physical constants of polycrystalline materials. Soviet Physics - Doklady, 11:323-325, 1966.

[4] T. Böhlke. Crystallographic Texture Evolution and Elastic Anisotropy: Simulation, Modeling, and Applications. Aachen: Shaker Verlag, 2001. Dissertation, Fakultät für Maschinenbau, Otto-von-GuerickeUniversität Magdeburg. 
[5] T. Böhlke and A. Bertram. A minimum problem defining effective isotropic elastic properties. Z. angew. Math. Mech., 80(S2):S419-S420, 2000.

[6] T. Böhlke and A. Bertram. The evolution of Hooke's law due to texture development in polycrystals. Int. J. Solids Struct., 38(52):9437-9459, 2001.

[7] T. Böhlke, A. Bertram, and E. Krempl. Modeling nonlinear effects in free-end torsion. To appear in Int. J. Plast., 2003.

[8] H.-J. Bunge. Zur Darstellung allgemeiner Texturen. Z. Metallkde., 56:872-874, 1965.

[9] H.-J. Bunge and W.T. Roberts. Orientation distribution, elastic and plastic anisotropy in stabilized steel sheet. J. Appl. Cryst., 2:116-128, 1969.

[10] G.J. Davies, D.J. Goodwill, and J.S. Kallend. Elastic and plastic anisotropy in sheets of cubic metals. Met. Trans., 3:1627-1631, 1972.

[11] I.M. Gel'fand, R.A. Minlos, and Z.Ya. Shapiro. Representations of the Rotation and Lorentz Groups and their Applications. Oxford: Pergamon Press, 1963.

[12] M. Guidi, B.L. Adams, and E.T. Onat. Tensorial representation of the orientation distribution function in cubic polycrystals. Textures Microstruct., 19:147-167, 1992.

[13] R. Hill. A theory of yielding and plastic flow of anisotropic materials. Proc. Phys. Soc. Lond., A 193: 281-297, 1948.

[14] J.S. Kallend and G.J. Davies. The elastic and plastic anisotropy of cold-rolled sheets of copper, gilding metal, and $\alpha$-brass. J. Inst. Met., 5:257-260, 1971.

[15] C.-S. Man. On the correlation of elastic and plastic anisotropy in sheet metals. J. Elast., 39:165-173, 1995.

[16] C.-S. Man. On the constitutive equations of some weakly-textured materials. Arch. Rat. Mech. Anal., 143:77-103, 1998.

[17] S. Matthies and M. Humbert. On the principle of a geometric mean of even-rank symmetric tensors for textured polycrystals. J. Appl. Cryst., 28:254-266, 1995.

[18] A. Reuss. Berechnung der Fließgrenze von Mischkristallen auf Grund der Plastizitätsbedingung für Einkristalle. Z. Angew. Math. Mech., 9:49-58, 1929.

[19] R.J. Roe. Description of crystalline orientation of polycrystalline materials. III. General solution to pole figure inversion. J. Apll. Phys., 36:2024-2031, 1965.

[20] C.A. Stickels and P.R. Mould. The use of Young's modulus for predicting the plastic-strain ratio of low-carbon steel sheets. Met. Trans., 1:1303-1312, 1970.

[21] C. Teodosiu, editor. Plasticity of single crystals and crystalline aggregates. CISM Courses and Lectures. Springer-Verlag, 1996.

[22] R. v. Mises. Mechanik der plastischen Formänderung bei Kristallen. Z. angew. Math. Mech., 8(3): 161-185, 1928.

[23] W. Voigt. Lehrbuch der Kristallphysik. Teubner Leipzig, 1910.

[24] Q.-S. Zheng and Y.-B. Fu. Orientaion distribution functions for microstructures of heterogeneous materials: I Directional distribution functions and irreducible tensors. Appl. Math. Mech., 22(8):865$884,2001$.

[25] Q.-S. Zheng and Y.-B. Fu. Orientaion distribution functions for microstructures of heterogeneous materials: II Crystal Distribution functions and irreducible tensors restricted by various material symmetries. Appl. Math. Mech., 22(8):885-903, 2001. 